\title{
Peculiarities of Shape Recovery in Polymer Composites with Compacting Filler
}

\author{
V. A. Beloshenko and Yu. V. Voznyak \\ Donetsk Physics and Technology Institute, National Academy of Sciences of Ukraine, 72 R. Luxemburg Street, 83114 Donetsk, Ukraine \\ Correspondence should be addressed to Yu. V. Voznyak, voznyak@mail.ru
}

Received 16 October 2010; Revised 15 December 2010; Accepted 23 January 2011

Academic Editor: Xianglong Meng

Copyright ( $) 2011$ V. A. Beloshenko and Yu. V. Voznyak. This is an open access article distributed under the Creative Commons Attribution License, which permits unrestricted use, distribution, and reproduction in any medium, provided the original work is properly cited.

\begin{abstract}
Peculiarities of the shape memory effect development in composites based on the epoxy polymer and various fillers, such as thermoexpanded graphite, aerosils, metallized graphite, and basalt flakes, have been investigated. It has been determined that straining followed by the shape recovery of composites is accompanied by changes in their volume. Extent and character of the changes depend on the ability of fillers to compaction under pressure, deformation scheme, adsorption ability of the filler. It is shown that the combined deformation consisting of compression and stretching of specimens in different sequence gives structural states for which the longitudinal strain-transverse strain ratio can take zero, positive, or negative values.
\end{abstract}

\section{Introduction}

Recently, there has been much interest shown in the effect of shape recovery or, in other words, in the shape memory effect (SME) in polymer systems of different nature [14]. This is consistent with the tendency of intensively developing studies of the so-called "smart" materials with polymers possessing the SME among them. The effect can be formed by specimen deformation at a temperature close to the glass-transition temperature $T_{g}$ or melting temperature followed by fixation of the stressed state. Such an effect developing under heating is not, as a rule, accompanied by significant changes in volume. However, when a porous structure originates in the material, one can observe the process of the initial-shape recovery and the irreversible volume increase [5-7]. The increase in volume is due to the opening of microvoids that were healed during deformation. These requirements are, in particular, satisfied by polymer composites with a compacting filler.

Our investigations of the epoxy polymer-thermoexpanded graphite (EP-TEG) system $[6,7]$ have shown that the composites are very promising. Depending on a chosen scheme of preliminary deformation, it was possible to control the value of one of the geometric dimensions of the specimen upon heating. At the same time, the proposed physical model [4] admitted the preparation of specimens from a porous composite, and they were able to simultaneously increase their dimensions during the SME realization. The mentioned composite would behave like a material with negative Poisson's ratio. In our case, this is the coefficient of proportionality between longitudinal and transverse deformation. Such specimens were for the first time obtained from the EP-TEG composite by a combined deformation (plunger extrusion and compression in a mould) [6].

This paper is aimed at revealing regularities for the SME developed in polymer composites with a compacting filler under different loading schemes.

\section{Experimental}

In $[6,7]$, a hot-hardened epoxy composition was the basic one. And it was to some extent difficult to have specimens of uniform compositions because of sedimentation process. In this experiment, to reduce the effect, the polymer matrix was a cold-hardened composition based on epoxy resin and dicyanoethulated diethylenetriamine taken in a stoichiometric ratio. The fillers were TEG, aerosil with specific surface value of $175 \mathrm{~m}^{2} / \mathrm{g}$ (A-175), $300 \mathrm{~m}^{2} / \mathrm{g}$ (A-300) and $380 \mathrm{~m}^{2} / \mathrm{g}$ (A-380), coppered graphite with copper content of $69 \mathrm{wt} \%$ (C-69), basalt flakes (BF). The specimens were prepared by 
the mixing of epoxy-composition components and fillers in a required ratio followed by hardening in cylindrical moulds by the following schedule: $20^{\circ} \mathrm{C}, 8 \mathrm{hs}+80^{\circ} \mathrm{C}, 6 \mathrm{hs}$.

The TEG was produced by a procedure described in $[6,7]$, the coppered graphite by a procedure of [8]. Its dispersity was $50-800 \mu \mathrm{m}$. Aerosils were industrial products of corresponding grades. BF were produced as follows: basalt melt of the chemical compositions ( $w \mathrm{t} \%): \mathrm{SiO}_{2}-30.2 ; \mathrm{FeO}+$ $\mathrm{Fe}_{2} \mathrm{O}_{3}-16.1 ; \mathrm{TiO}_{2}-1.6 ; \mathrm{Al}_{2} \mathrm{O}_{3}-14.4 ; \mathrm{Ca} 0$ - 9.6; $\mathrm{MgO}-4.1$; $\mathrm{K}_{2} \mathrm{O}-3.2$ was passed through die orifice, thus forming a jet, the latter got to the rotating turbine of a distributing device. Film flowing out of the rotating turbine was broken by the flow of compressed air. The resulting were basalt flakes of $30 \mu \mathrm{m}-2 \mathrm{~mm}$ diameter and nearly $3 \mu \mathrm{m}$ thick.

The cured billets with a diameter of $7-10 \mathrm{~mm}$ and a length of $20 \mathrm{~mm}$ were deformed in the high-elastic state and then cooled under isometric conditions to $T \prec T_{g}$. Shape recovery was attained by the heating in the free state to $T=85^{\circ} \mathrm{C}$ (which is higher than $T_{g}$ ) and by storing for $15 \mathrm{~min}$. The deformation was implemented on a universal testing machine by the plunger extrusion, compression in a mould and by combining the plunger extrusion to a die with elongated channel and the compression in a cylindrical mould of diameter equal to that of die channel.

The strains during extrusion $\varepsilon_{e}$ and compression $\varepsilon_{c}$ were calculated by the formulas $\varepsilon_{e}=\left(d-d_{e}\right) / d\left(d, d_{e}\right.$ : sample diameters before and after extrusion) and $\varepsilon_{c}=\left(l-l_{c}\right) / l(l$, $l_{c}$ : sample length before and after compression). In the case of combined deformation, the axial and radial logarithmic strains were estimated by the formulas: $\varepsilon_{l}=\ln \left(l / l_{0}\right)$ and $\varepsilon_{r}=\ln \left(r / r_{0}\right)$, where $l_{0}, l, r_{0}, r$ are the initial and current length and radius of the cylindrical specimen, respectively. The residual strain upon the recovery after compression was found as $\varepsilon_{\text {res }}=\left(l-l_{\text {rec }}\right) / l$, where $l, l_{\text {rec }}$ : length of initial and recovered specimen, upon the recovery after extrusion: $\varepsilon_{\text {res }}=\left(d-d_{\text {rec }}\right) / d$, where $d, d_{\text {rec }}$ : diameter of initial and recovered specimen.

The bulk density of fillers was determined by a standard method, that is, the weighing of powder in a measuring cylinder of a known volume. The density of compacted powders was determined by weighing and by measuring the geometric dimensions of cylindrical specimens. The density of composites $\theta_{c}$ was found by the hydrostatic weighing method using a scales of the AX200 type, Shimadzu company.

$T_{g}$ was determined by the methods of differential scanning calorimetry on a thermoanalytical complex DuPont 9900 . The specimens were heated at a rate of $20 \mathrm{deg} / \mathrm{min}$, the mass of weight $-15 \mathrm{mg}$.

Uniaxial compression tests were done on a universal testing machine, the specimens were $15 \mathrm{~mm}$ high with the diameter of $10 \mathrm{~mm}$. The velocity of supporting platform travel was equal to $0.5 \mathrm{~mm} / \mathrm{min}$. Microhardness $H_{\mu}$ was measured by indenter load of $0.5 \mathrm{~N}$. The $H_{\mu}$ value was the average of 15-20 measurements.

The strains developed in the specimen under heating in isometric conditions were measured using a universal testing machine. For specimens of diameter $10 \mathrm{~mm}$ and height $15 \mathrm{~mm}$ the rate of heating was equal to $1 \mathrm{deg} / \mathrm{min}$.
The microstructure was investigated and microphotography was done using a light reflection microscope. The structure was examined at the specimen polished end faces with no etching. The "Image-Tool" and "Excel" application package was used to process the results of quantitative analysis of microstructure whose image was fixed by digital electronic camera.

TEM observation were performed on a JEM-200A Jeol transmission electron microscope; the accelerating voltage was $200 \mathrm{kV}$. Replicas from the fractured surface were prepared as follows. The X-ray film (matrix) was treated with acetone and glued directly onto the fractured surface. Then, the film was dried, and the dried pattern was decorated with a thin carbon layer. The carbon replica was separated from the matrix and studied by TEM.

\section{Results and Discussion}

Deformation of polymer composites by means of extrusion and compression in a mould was chosen by us because of several reasons. First, these are comparatively simple technological methods to get order in orientation. Second, there is the difference in the scheme of developed stressed-strained state of the material. In the case of extrusion the strain tensor has two negative $\left(\varepsilon_{r r}, \varepsilon_{\varphi \varphi} \prec 0\right)$ and one positive $\left(\varepsilon_{z z} \succ 0\right)$ components. In the case of compression in a mould-two zero $\left(\varepsilon_{r r}=\varepsilon_{\varphi \varphi}=0\right)$ and one negative $\left(\varepsilon_{z z} \prec 0\right)$ components. As a result, in the deformed specimen, there are stresses different in sign: after the extrusion compressive and after the compression-tensile ones. Third, these methods of plastic working give cylindrical specimens with which it is possible to implement two variants of combined deformation, such as extrusion compression in a mould and compression in a mould-extrusion. With the mentioned differences in deformation schemes taken into account it is appropriate to consider peculiarities of the SME for each case separately.

3.1. Compression in a Mould. From data of Table 1 it is seen that with the increase in filler concentration $C$ the value of the maximum attainable strain $\varepsilon_{\max }$, with no composite failure, varies extremely for all the investigated fillers. And the absolute values of $\varepsilon_{\max }$ and the position of maximum on the $\varepsilon_{\max }(C)$ dependence are, to a much extent, determined by filler type. The heating of deformed specimens to $T>T_{g}$ is accompanied by shape recovery (increase of the length with the diameter remained unchanged); however, for same values of $C$, the process becomes partially irreversible. Value of residual strain and conditions of its origination depend, the same as in the case of $\varepsilon_{\max }$, on filler type.

The obtained results imply that a universal parameter need be introduced to describe the behavior of composite under filler type variation. As these materials are capable of changing their volume upon deformation and shape recovery due to the presence of microvoids, it seems natural to pay attention to the initial (bulk) density $\theta$ of the filler and to its tendency to compaction under pressure. The latter can be estimated by the relative density $\theta_{\text {rel }}$ equal to the ratio of bulk density of the powder to density of the compact. 
TABLE 1: Characteristics of the EP and EP-based composites (compression in a mould).

\begin{tabular}{|c|c|c|c|c|c|}
\hline Composite & $C, \mathrm{wt} \%$ & $\theta_{c}, \mathrm{~g} / \mathrm{cm}^{3}$ & $T_{g},{ }^{\circ} \mathrm{C}$ & $\mathcal{E}_{\max }$ & $\varepsilon_{\text {res }}$ \\
\hline $\mathrm{EP}$ & 0 & 1.0020 & 50 & - & - \\
\hline \multirow{4}{*}{ EP-TEG } & 10 & 1.0926 & 51 & 0.20 & 0 \\
\hline & 15 & 1.1323 & 57 & 0.30 & 0 \\
\hline & 20 & 1.1738 & 63 & 0.45 & 0 \\
\hline & 25 & 1.1816 & 65 & 0.30 & 0.028 \\
\hline \multirow{3}{*}{ EP-A-175 } & 5 & 1.0489 & 58 & 0.07 & 0 \\
\hline & 10 & 1.1290 & 60 & 0.26 & 0 \\
\hline & 15 & 1.1779 & 62 & 0.22 & 0.057 \\
\hline \multirow{3}{*}{ EP-A-300 } & 5 & 1.0494 & 60 & 0.08 & 0 \\
\hline & 10 & 1.141 & 65 & 0.27 & 0 \\
\hline & 15 & 1.1907 & 67 & 0.23 & 0.052 \\
\hline \multirow{3}{*}{ EP-A-380 } & 5 & 1.0498 & 62 & 0.10 & 0 \\
\hline & 10 & 1.1790 & 66 & 0.30 & 0 \\
\hline & 15 & 1.2283 & 68 & 0.26 & 0.045 \\
\hline \multirow{3}{*}{ EP-C-69 } & 10 & 1.2612 & 52 & 0.10 & 0 \\
\hline & 20 & 1.4623 & 57 & 0.15 & 0 \\
\hline & 30 & 1.5422 & 61 & 0.12 & 0.040 \\
\hline \multirow{4}{*}{ EP-BF } & 10 & 1.2438 & 59 & 0.04 & 0 \\
\hline & 20 & 1.2669 & 61 & 0.05 & 0 \\
\hline & 30 & 1.3072 & 64 & 0.06 & 0 \\
\hline & 40 & 1.3445 & 67 & 0.05 & 0.035 \\
\hline
\end{tabular}

Figure 1 shows dependences of $\varepsilon_{\max }$ on parameters $\theta_{\text {rel }}$ and $\theta$ for the investigated fillers. The maximum values of $\varepsilon_{\max }$ were taken from Table 1 . In the first case, there is a monotonous decrease of $\varepsilon_{\max }$ with the increase of relative density. The dependence is analytically described by a simple logarithmic function

$$
\varepsilon_{\max }=-10.5 \ln \theta_{\text {rel }}-2.9,
$$

making it possible to predict the behavior of composite, with $\theta_{\text {rel }}$ known, during the development of the SME. In its turn, the relationship between $\varepsilon_{\max }$ and $\theta$ is ambiguous, that is, the bulk density can not be used for finding a correlation with value of the maximum strain.

The extremum change of $\varepsilon_{\max }$ with the increase of filler content in the composite (Table 1 ) is explained by competition of two processes. The basic polymer is not practically compressed in the mould because the free volume is small. The introduction of filler results in origination of microvoids making the process of plastic deformation easier. This is also accompanied by volume decrease and growth of $\varepsilon_{\max }$. The presence of filler results in the origination of interfacial surface and in the formation of boundary layers [9]. Such adsorbed layers possess high enough density, so the increase of their summary volume with $C$ growth results in the decreasing of deformability of the composite. When, for

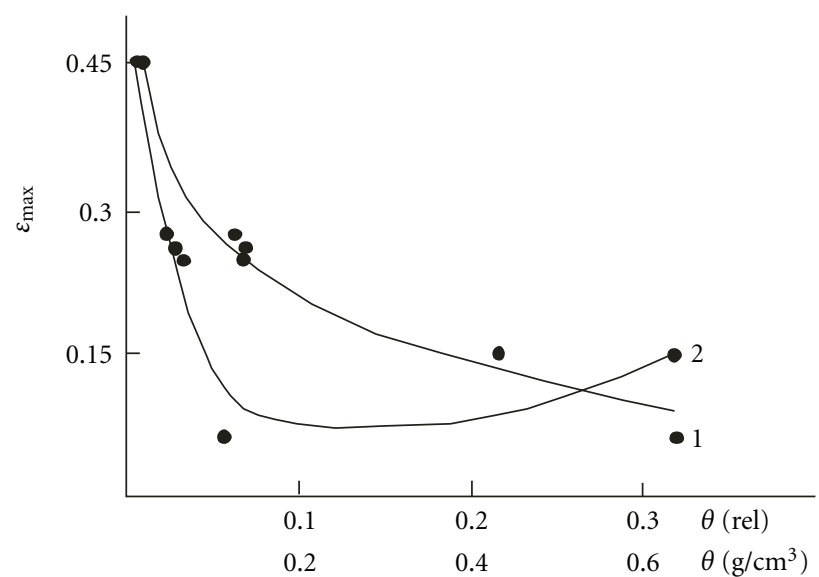

FIGURE 1: Dependence of the maximum strain of composites compressed in a mould on relative density (1) and bulk density (2) of fillers.

some values of $C$, the second process is prevailing, the $\varepsilon_{\max }$ value becomes decreasing.

The introduction of fillers increases $T_{g}$ as compared to the basic polymer (Table 1) and it should be added that the effect increases with filler content and with value of its specific surface. This is confirmed by the data obtained for aerosil. This result agrees well with the known representations [9], it is related to deceleration of segmental mobility in boundary layers.

The origination of residual strain (Table 1) is, probably, related to the achievement of a specific concentration of boundary layers, when the original dimensions of specimens do not recover completely because of the limited molecular mobility. Another possible reason responsible for $\varepsilon_{\text {res }}$ is a partial mechanical destruction of polymer network occurring under high deformations and rich filling.

Differences in $\varepsilon_{\text {res }}$ magnitudes are to some extent due to different adsorption interaction between polymer and filler. For the EP-TEG system the interaction is much weaker than for the rest polymer composites [9]. Hence, $\varepsilon_{\text {res }}$ is the least. It should be, however, noted that the mechanism responsible for the occurrence of the residual strain could not be only reduced to the adsorption interaction in boundary layers. This is evidenced by the decrease of $\varepsilon_{\text {res }}$ observed during the growth of the specific surface of aerosil and by the expected enhancement of the adsorption interaction. There are evidently several competing processes, such as changes in ratios of densities of chemical and physical angles in boundary layers [9].

With the growth of filler concentration the density of composites is increasing (Table 1). But for different materials, there are different reasons of $\theta_{c}$ behavior. In Figure 2, there are the results of measurements of the composite density represented by diverging curves standing for experimental values and those calculated by the additivity principle. As it is somewhat difficult to estimate the amount of fillers which is composite's component in the loose and compacted states, for a correct estimation, we took the experimental densities of specimens in the compressed state, while in calculations we used densities of EP and filler in the compacted state. 


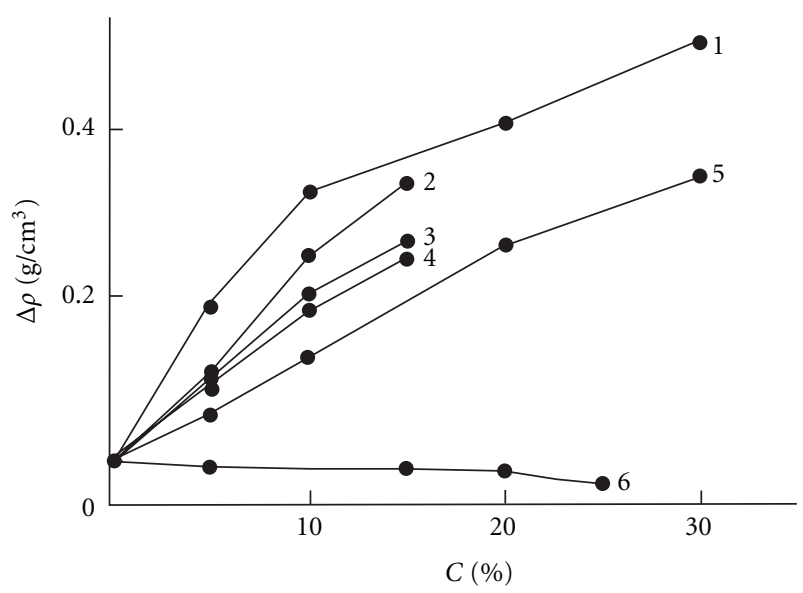

Figure 2: Dependence of the difference between experimental and theoretical values of the density of composites on filler type and content: (1) BF; (2) A-380; (3) A-300; (4) A-175; (5) C-69; (6) TEG.

In the case of composites containing aerosil, $\mathrm{BF}$ and C69, the experimental data turned out to be higher than the calculated ones, thus evidencing that the transient layers have been formed with a density higher than that of the polymer matrix. This is connected with a high surface energy of the mentioned fillers. For composites with TEG the calculated values of $\theta_{c}$ are higher than the experimental ones because of the presence of loose boundary layers. An increase in the density of the EP-TEG composites (Table 1) is attained only at the expense of higher density of the filler as compared to the polymer matrix.

It is interesting to compare the data of Table 1 with the results of uniaxial compression and microhardness test (Table 2). They correlate with each other to a considerable extent. The addition of aerosil to the EP hardens the material and this is indicated by values of the yield strength $\sigma_{y}$, modulus of elasticity $E$ and microhardness $H_{\mu}$. The largest changes are noted for A-380. At the same time, the plasticity of composites becomes lower and the change is sizable for the composite of larger specific surface. This is in accordance with representations on the existence of boundary layers. Only the yield strain $\varepsilon_{y}$ for $C=15 \mathrm{wt} \%$ deviated from the general behavior. These facts show that the processes taking place in the composite under filling are complex.

In the case of the EP-TEG composite, the disordering of the material was observed together with the increase of plasticity. This points to a weak adsorption interaction between TEG and the epoxy matrix because of the low surface energy of graphite.

The results of electron microscopic studies also agree with the data listed in Tables 1 and 2. Figure 3 presents microscope images of the fracture surface of composites containing TEG, A-175 and A-380. In the case of the EP-TEG composite (Figure 3(a)), the fractured surface passes through the interfacial boundary; on approaching the TEG surface, the structure of the polymer matrix does not change and this pattern is associated with the weak interaction between a polymer and filler.
TABLE 2: Mechanical properties of the EP and EP-based composites.

\begin{tabular}{lccccc}
\hline Composite & $C, \mathrm{wt} \%$ & $H_{\mu}, \mathrm{MPA}$ & $\sigma_{y}, \mathrm{MPa}$ & $\varepsilon_{y}, \%$ & $E, \mathrm{MPa}$ \\
\hline EP & & 120 & 80 & 9.0 & 705 \\
& 10 & 72 & 65 & 10.4 & 676 \\
EP-TEG & 15 & 70 & 58 & 10.6 & 616 \\
& 20 & 68 & 48 & 11.8 & 564 \\
& 25 & 64 & 46 & 12.1 & 556 \\
\hline \multirow{3}{*}{ EP-A-175 } & 5 & 126 & 100 & 7.8 & 778 \\
& 10 & 135 & 106 & 7.6 & 804 \\
& 15 & 146 & 114 & 7.2 & 820 \\
\hline \multirow{3}{*}{ EP-A-300 } & 5 & 132 & 105 & 7.6 & 799 \\
& 10 & 138 & 116 & 7.3 & 850 \\
& 15 & 149 & 124 & 7.0 & 878 \\
\hline \multirow{2}{*}{ EP-A-380 } & 5 & 134 & 110 & 7.4 & 814 \\
& 10 & 143 & 123 & 7.3 & 894 \\
& 15 & 162 & 132 & 7.2 & 950 \\
\hline
\end{tabular}

For composites containing aerosil (A-175 and A-380), a crack propagates across the polymer matrix (Figures $3(\mathrm{~b})$ and 3(c)) and this indicates the existence of a strong adsorption interaction in the boundary layer. In this case, in the composites containing a filler with a high specific surface (A-380), a more energy consuming interfacial boundary is formed, and hence, the adsorption interaction is enhanced.

Figure 4 illustrates changes in stresses $\sigma_{\text {rec }}$ developed in the composites under shape recovery. The heating of specimens under the isometric conditions results in the occurrence of $\sigma_{\text {rec }}$ maxima in the region of glass-transition temperatures. Their height depends on filler properties and magnitude of specific surface. In the EP-TEG composite, near $60^{\circ} \mathrm{C}$, comparatively low stresses are generated. In the case of the EP-aerosil system, the stresses are higher, especially in materials based on EP and A-380. The correlation between $\sigma_{\text {rec }}$ and strength characteristics of the composites is evident, that is the hardening contributes to generation of internal stresses when shape recovery is constrained. The obtained result is in agreement with conclusions of paper $[10,11]$ where the authors studied the SME in the EP-SiC system.

In such a way, taking the polymer matrix of high deformability and the compacting filler of low relative density, it is possible to obtain composites for products which will recover their shape with the maximum change in volume. The SME characteristics can be also controlled by using fillers of various surface energies.

3.2. Extrusion. In the case of extrusion, both the matrix polymer and the composites with different filler content make it possible to realize high enough strain with no specimen failure. Table 3 lists data of the experiments done on EP and systems of EP-TEG, EP-aerosil. The heating of extrudates to $T \succ T_{g}$ is accompanied by recovery of the original shape at the expense of diameter increase and length 


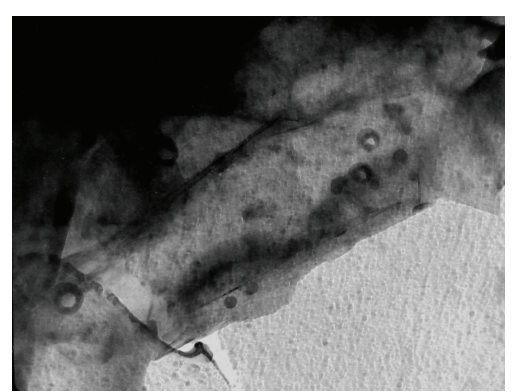

(a)

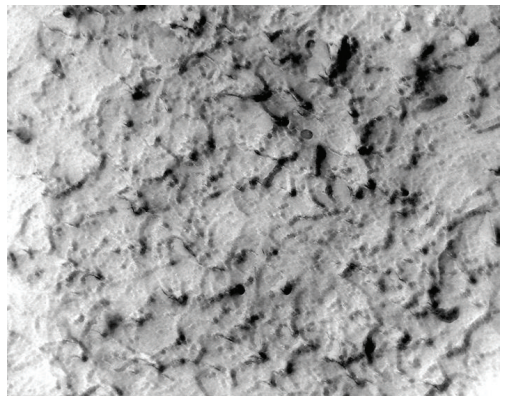

(b)

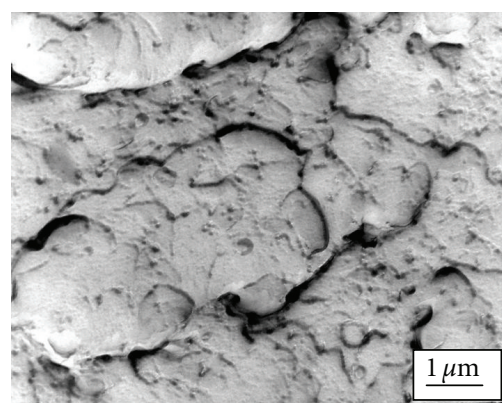

(c)

Figure 3: Microscopic images of the fractured surface for the composites under study: (a) EP-TEG, (b) EP-A-175, and (c) EP-A-380. Hereinafter, filler content is $10 \mathrm{wt} . \%$.

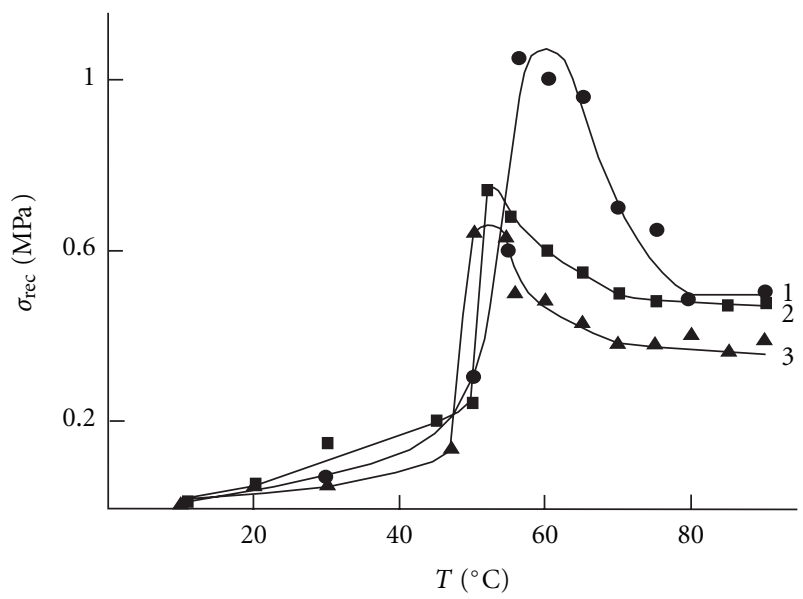

Figure 4: Temperature dependences of stresses developed in specimens of the composites upon shape recovery: (1) EP-A-380 and (2) EP-A-175 and (3) EP-TEG. $\varepsilon_{c}=0.25$.

contraction. In this case, in contrast to compression in the mould, the residual deformation is conserved already at low concentrations of filler. Its value depends on both the filler type and the degree of stretching. Under the extrusion, $\varepsilon_{\text {res }}$ for the EP-TEG is lower than for other materials, and for all the composites it increases with $\varepsilon_{e}$ and $C$.

The comparison of results obtained for aerosils of three types, differing in the magnitude of specific surface, shows the following. There is a little difference in absolute values of $\varepsilon_{\text {res }}$ corresponding to one and the same values of $C$ and $\varepsilon_{e}$. Nevertheless, there is a clear tendency of $\varepsilon_{\text {res }}$ decrease in the $\mathrm{A}-175 \rightarrow \mathrm{A}-300 \rightarrow \mathrm{A}-380$ series. It is seen the best for the maximum values of $\varepsilon_{e}$. The revealed regularity correlates with the found in the case of compression (Table 1): an increase in the specific surface of filler decreases $\varepsilon_{\text {res }}$. The like good agreement is in the case of polymers filled with graphite. In the polymers, $\varepsilon_{\text {res }}$ is lower than in EP-aerosil composites both in the case of extrusion and compression. This difference becomes more considerable when the comparison is more correct, that is, when filler content is evaluated in volume percent, not in the weight ones.
The absence of residual deformation after the recovery of extruded matrix-polymer shape underlies the decisive contribution of boundary layers to the formation of $\varepsilon_{\text {res }}$. Resting on this fact, one can presuppose the following mechanism of residual strain development in composites subjected to plunger extrusion. When the material is extruded through the die, the deformation of elongation plays the dominant part in the stressed-strained state scheme. Its action results in a more intensive, than under compression, failure of the intersite fragments of the polymer network in boundary layers, and the shape recovery becomes irreversible. In composite with weak physical bonds between filler and polymer matrix (EP-TEG), the polymer chains are very mobile in boundary layers and this conditions a lower probability of failure (lower values of $\varepsilon_{\text {res }}$ ) as compared to systems with strong adsorption interaction (EP-aerosil). It is, at the same time, known [9] that in the filler the density of chemical crosslinking on the surface is lower than far from the boundary layer. Changing the mobility of intersite chains, this factor influences the tendency of the material to failure. In the case of aerosil with the most developed surface (A-380), the factor provides better conditions for preserving the integrity of polymer network during the deformation by extrusion and compression in the mould.

Consequently, for the formation of the SME in composites based on network polymers by means of solid-phase extrusion, one should remember that the presence of filler can't guarantee the complete shape recovery. This peculiarity of the SME is connected with the scheme of the stressedstrained state of the material and provoked by the failure of polymer-network elements near filler surface occurring under deformation.

3.3. Combined Deformation. As revealed on the example of simple loading schemes (Figure 1 and Table 3), in the filled network polymer the maximum strains are attained in composites with fillers of low relative density. That is why, for the combined deformation, we took composites containing TEG and aerosil.

To reduce the bulk of work, we varied the value of $C$ and limited ourselves by filler content of $10 \mathrm{wt} \%$. This is the 
TABLE 3: Influence of composition and degree of deformation of composites during extrusion of the ability of shape recovery.

\begin{tabular}{|c|c|c|c|}
\hline Composite & $C, \mathrm{wt} \%$ & $\varepsilon_{e}$ & $\varepsilon_{\text {res }}$ \\
\hline \multirow{3}{*}{$\mathrm{EP}$} & \multirow{3}{*}{0} & 0.1 & 0 \\
\hline & & 0.2 & 0 \\
\hline & & 0.3 & 0 \\
\hline \multirow{9}{*}{ EP-TEG } & \multirow{2}{*}{10} & 0.1 & 0.011 \\
\hline & & 0.2 & 0.024 \\
\hline & \multirow{4}{*}{15} & 0.3 & 0.028 \\
\hline & & 0.1 & 0.022 \\
\hline & & 0.2 & 0.036 \\
\hline & & 0.3 & 0.067 \\
\hline & \multirow{3}{*}{20} & 0.1 & 0.032 \\
\hline & & 0.2 & 0.059 \\
\hline & & 0.3 & 0.125 \\
\hline \multirow{9}{*}{ EP-A-175 } & \multirow{3}{*}{25} & 0.1 & 0.062 \\
\hline & & 0.2 & 0.091 \\
\hline & & 0.3 & 0.147 \\
\hline & \multirow{2}{*}{5} & 0.1 & 0.002 \\
\hline & & 0.2 & 0.020 \\
\hline & \multirow{4}{*}{10} & 0.3 & 0.054 \\
\hline & & 0.1 & 0.020 \\
\hline & & 0.2 & 0.030 \\
\hline & & 0.3 & 0.057 \\
\hline \multirow{9}{*}{ EP-A-300 } & \multirow{3}{*}{15} & 0.1 & 0,027 \\
\hline & & 0.2 & 0.040 \\
\hline & & 0.3 & 0.074 \\
\hline & \multirow{3}{*}{5} & 0.1 & 0.002 \\
\hline & & 0.2 & 0.020 \\
\hline & & 0.3 & 0.045 \\
\hline & \multirow{3}{*}{10} & 0.1 & 0.020 \\
\hline & & 0.2 & 0.028 \\
\hline & & 0.3 & 0.050 \\
\hline \multirow{12}{*}{ EP-A-380 } & \multirow{3}{*}{15} & 0.1 & 0.027 \\
\hline & & 0.2 & 0.040 \\
\hline & & 0.3 & 0.072 \\
\hline & \multirow{3}{*}{5} & 0.1 & 0.002 \\
\hline & & 0.2 & 0.024 \\
\hline & & 0.3 & 0.042 \\
\hline & \multirow{3}{*}{10} & 0.1 & 0.020 \\
\hline & & 0.2 & 0.028 \\
\hline & & 0.3 & 0.046 \\
\hline & \multirow{3}{*}{15} & 0.1 & 0.027 \\
\hline & & 0.2 & 0.038 \\
\hline & & 0.3 & 0.072 \\
\hline
\end{tabular}

situation when with the both methods of specimen preparation we expect rather high values of $\varepsilon_{\max }$ with the minimization of $\varepsilon_{\text {res. }}$. This statement is based on data of Tables 1 and 3 .
It has been shown [12] that it is convenient to represent the processes of deformation and shape recovery within the coordinates of logarithmic strain-the axial $\varepsilon_{l}$ and the radial $\varepsilon_{r}$. Figure 5(a) illustrates strain for the EP-TEG composite under the extrusion combined with the compression in the mould. Diameter of the circle is a measure of reversibility: the smaller the diameter, the closer the dimensions of heated composite to the original ones. The magnitude $\varepsilon=\varepsilon_{l}+2 \varepsilon_{r}$ characterizes changes in volume [12]. If the deformation of material occurs with no volume change, then $\varepsilon=0$, with a decrease in volume: $\varepsilon \prec 0$, with an increase in volume: $\varepsilon \succ 0$.

By analogy with Poisson's ratio $\nu$, for the case of high inelastic strains, we can introduce the effective coefficient of transverse strain $\nu_{\mathrm{ef}}$ by using the formula:

$$
\varepsilon_{r}=-\nu_{\mathrm{ef}} \varepsilon_{l} .
$$

However, in contrast to $v$, it is not a constant and depends on loading and unloading (shape recovery) conditions. It follows from the above relationship that in the I and III quadrants of the coordinate system $\left(\varepsilon_{l}, \varepsilon_{r}\right)$ parameter, $\nu_{\text {ef }}$ takes negative values, in II and IV quadrants-positive ones.

Figure 5(b) shows that when deformation of the composite includes extrusion followed by compression in a container with diameter of the channel equal to that of the extrudate, the extrusion gives positive values of $\nu_{\mathrm{ef}}$ of the specimen. Subsequent compression gives negative values of the coefficient if stretching is not large. The extent of recovery decreases with the increase of stretching extent.

In the case of the reverse scheme ("compression-extrusion"), the compressed specimen possesses zero $\nu_{\text {ef }}$ (shape recovery is by length increase with the diameter remaining unchanged), then the extrusion results in specimen state with negative $v_{\mathrm{ef}}$, and when the extent of stretching is large, $v_{\mathrm{ef}}$ is positive.

In the case of aerosol, the behavior of the trajectories of straining becomes somewhat different (Figure 6). In the case of combined deformation by the extrusion compression scheme, the subsequent compression gives (even for a large extent of the stretching) material with negative $\nu_{\text {eff }}$. This peculiarity extends to the reverse scheme: for $\varepsilon_{e}=0.2$ the composite EP-A-175 possesses negative $v_{\mathrm{ef}}$. With equal values of corresponding strains, a lower extent of the recovery, as compared to the EP-TEG system, is with specimens filled with aerosil. Moreover, the extent of recovery increases with the growth of specific surface value. This result correlates with the behavior of $\varepsilon_{\text {res }}$ in the case of simple schemes of loading (Tables 2 and 3 ). The extent of recovery depends on the utilized variant of combined deformation too. With the "extrusion compression" scheme, the composites show better capability to shape recovery.

As has been already mentioned, the change of composite volume under deformation and shape recovery is related to the ability of polymer matrix of "entering" and "leaving" the pores under the action of external stresses or internal stresses created in the material. This process is reflected in the microstructure of the filled systems. Studies of structure illustrating the changes typical of combined scheme of loading have been done with the EP-TEG composite, for which the deformation trajectories have been analysed (Figure 5). 


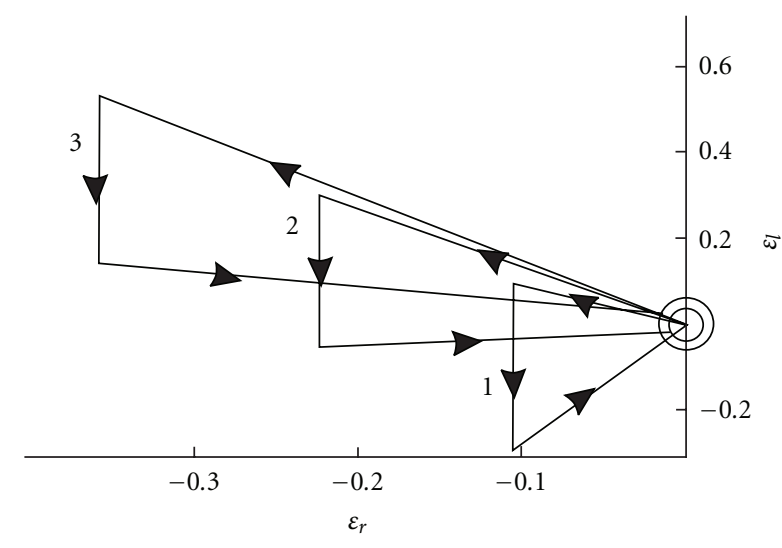

(a)

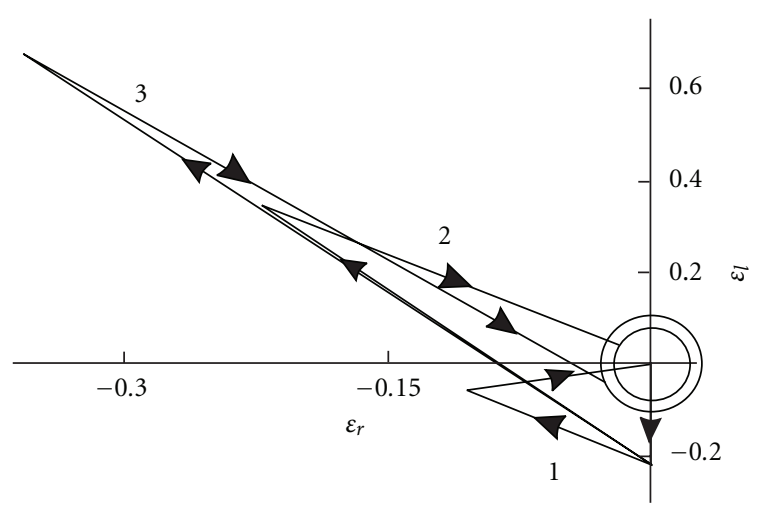

(b)

FIGURE 5: Trajectories of straining for EP-TEG composite by the routes: (a) extrusion compression shape recovery. (1) $\varepsilon_{e}=0.1$, $\varepsilon_{c}=0.31$; (1) $\varepsilon_{e}=0.2, \varepsilon_{c}=0.3$; (1) $\varepsilon_{e}=0.3, \varepsilon_{c}=0.48$. (b) compression-extrusion shape recovery. (1) $\varepsilon_{c}=0.2, \varepsilon_{e}=0.1$; (2) $\varepsilon_{c}=0.2, \varepsilon_{e}=0.2$; (4) $\varepsilon_{c}=0.2, \varepsilon_{e}=0.3$.

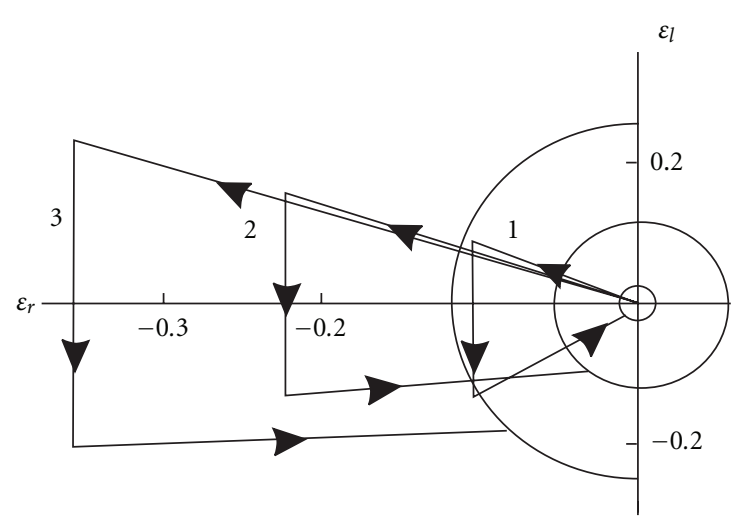

(a)

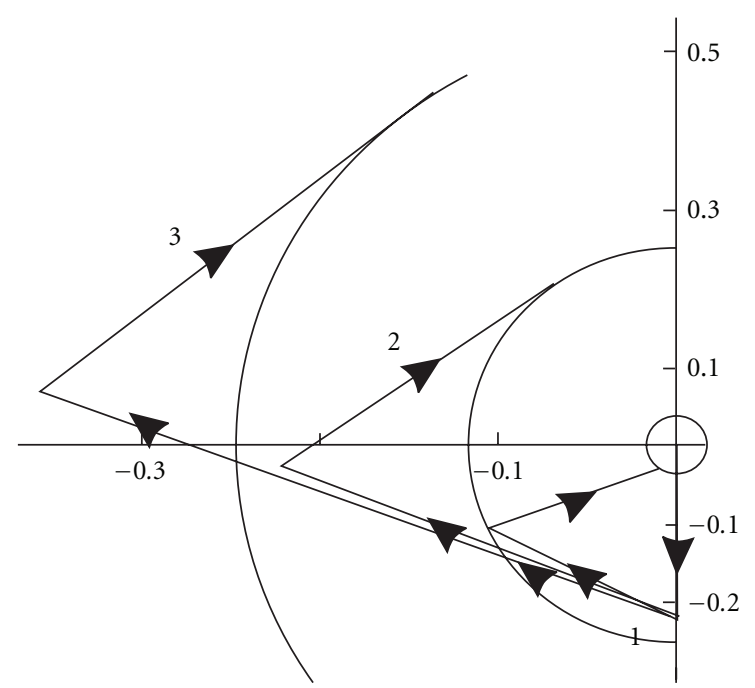

(b)

FIGURE 6: Trajectories of straining for EP-A-175 composite by the routes: (a) extrusion compression shape recovery. (1) $\varepsilon_{e}=0.1, \varepsilon_{c}=0.2$; (2) $\varepsilon_{e}=0.2, \varepsilon_{c}=0.25$; (3) $\varepsilon_{e}=0.3, \varepsilon_{c}=0.36$. (b) compression-extrusion shape recovery. (1) $\varepsilon_{c}=0.2, \varepsilon_{e}=0.1$; (2) $\varepsilon_{c}=0.2, \varepsilon_{e}=0.2$; (3) $\varepsilon_{c}=0.2, \varepsilon_{e}=0.3$.

To minimize the effect of material heterogeneity when performing comparative quantitative estimations, the specimens for different schemes of combined deformation were cut from the middle portion of one and the same cylindrical billet. The microstructure was studied on cuts made on interfaces.

It has been determined that in the original samples there are rather coarse aggregates of graphite particles of the average size $d=740 \mu \mathrm{m}$ (Figure $7(\mathrm{a})$ ) and $d=710 \mu \mathrm{m}$ (Figure 8(a)). Deformation by the "extrusion compression" scheme (Figure 7(b)) results, after the extrusion, in crushing and compaction of the aggregates to $=510 \mu \mathrm{m}$. Subsequent compression in the mould result in a higher compaction of filler aggregates $-d=420 \mu \mathrm{m}$ (Figurer 7(c)). The heating to $T \succ T_{g}$ in the free state results in recovery of the initial sample shape and enlargement of the average size of cavities filled by graphite to $700 \mu \mathrm{m}$ (Figure $7(d))$.

The dynamics of changes is well seen on the diagram of Figure 9(a).

Under the "compression-extrusion" scheme, the TEG aggregates first become refined and compacted with their initial size decreasing to $d=470 \mu \mathrm{m}$ (Figure 8(b)). This parameter is negligibly increased to $d=485 \mu \mathrm{m}$ after the extrusion (Figure 8(c)). In the recovered sample, $d=$ $680 \mu \mathrm{m}$ (Figure 8(d)). Thus, the character of microstructure evolution is different under different schemes of combined deformation.

Figure 9(b) illustrates the results of density measurements for the studied samples made after every stage of 


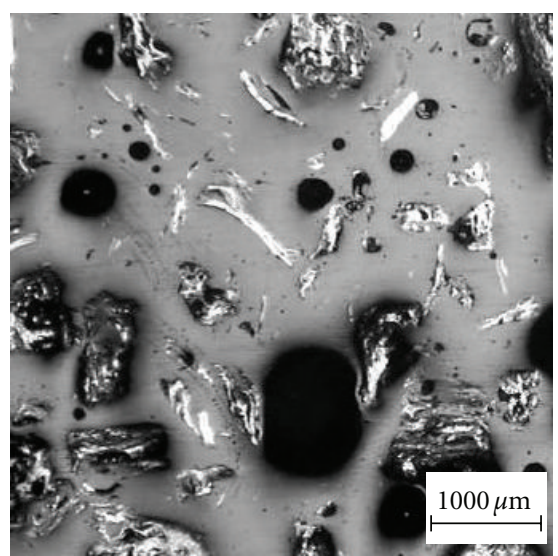

(a)

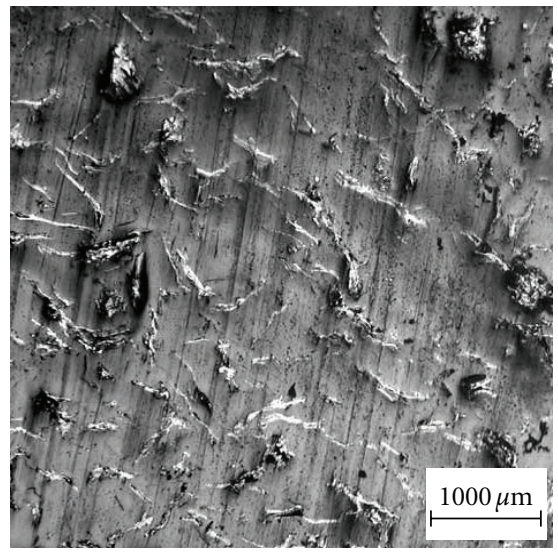

(c)

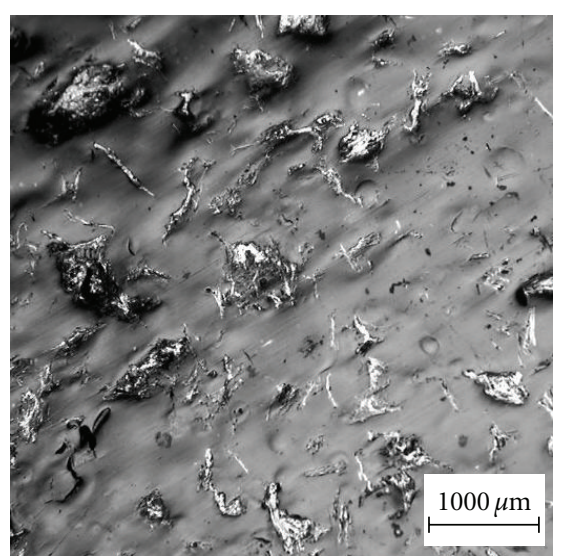

(b)

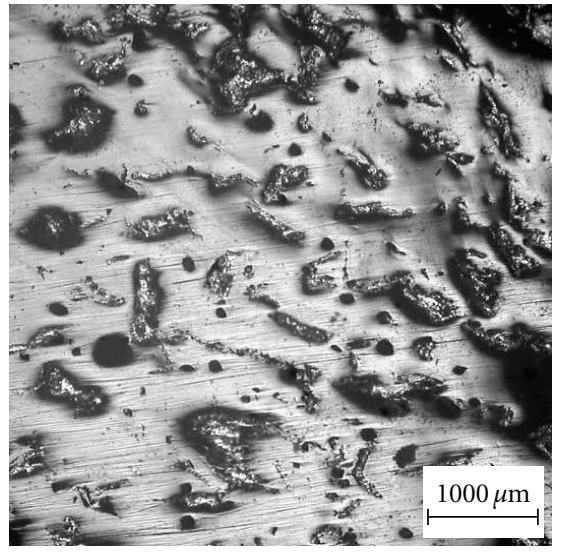

(d)

FIGURE 7: Surface micrographs of the EP-TEG (transverse section): (a) initial stage, (b) after extrusion, (c) after combined deformation, and (d) after combined deformation and shape recovery.

deformation. They correlate well with the data of Figure 9(a): the density of composite changes in the antibate way with the average size of graphite aggregates (the cavities filled with TEG). Changes in densities of samples resulting from each stage of deformation have been also analysed for the EPaerosil composites, see Table 4 . Here $\theta_{c}^{\text {init }}, \theta_{c}^{e}, \theta_{c}^{c}, \theta_{c}^{\text {rec }}$-density of initial composite, density after extrusion, compression and shape recovery, respectively.

The diagrams of Figure 9 as well as the data of Table 4 permit us to draw a number of conclusions, in particular, about the behavior of microvoids and the effective healing there of under different types of deformation.

Allowing for a high compressibility of the TEG [6] it can be assumed that already at the first stage of deformation the filler becomes compacted irrespective of the scheme of combined deformation utilized. Further its density varies a little even during the shape recovery. The increase of $d$ observed upon heating the composites is due to the opening of pores healed upon deformation when the combined deformation ends in compression, $d$ and $\theta_{c}$ change the most as compared to the original material. This is due to the decrease of volume occupied by the pores. Consequently, this type of combined deformation should be of preference when it is desirable to realize the SME with the largest changes in volume.

In such a way, the usage of combined deformation involving the processes of compression and tension in different sequence makes it possible to form such structural states of the composites when longitudinal-to-transverse deformation relationship may have, under shape recovery, both positive and negative values. The residual deformation under shape recovery is due to boundary layers present in the polymer composite.

The residual deformation value depends on both the adsorption ability of the filler and the deformation scheme.

Consider the mechanisms of SME formation and realization in crosslinked composites, including the filled ones. In the oriented polymers there are directions along which the segments of molecular chains align, this contrasts with nonoriented polymers or isotropic materials in which the macroscopic order is absent. The degree of molecular orientation depends on chemical structure and peculiarities of polymer structure organization, as well as on the method by which the orientation order is formed [2]. 


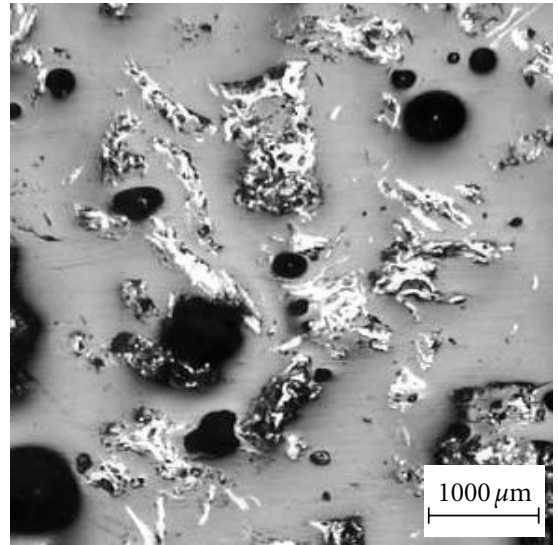

(a)

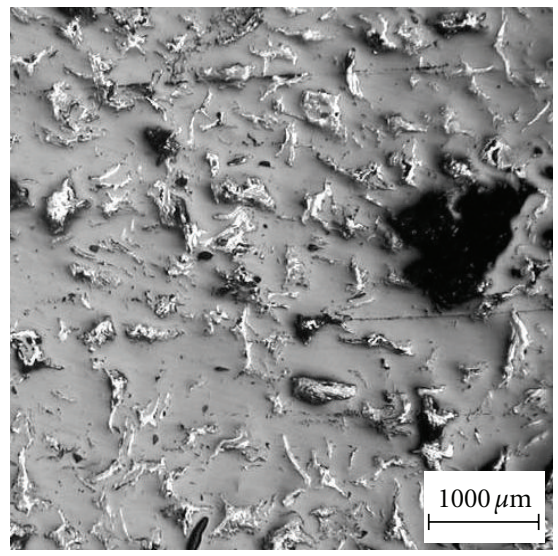

(c)

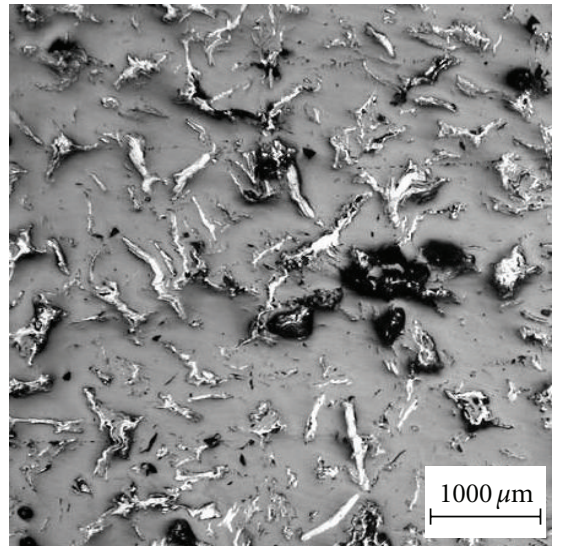

(b)

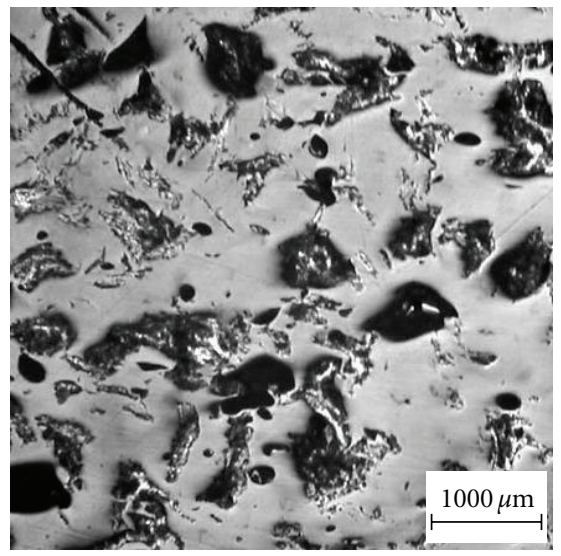

(d)

FIGURE 8: Surface micrographs of the EP-TEG (transverse section): (a) initial stage, (b) after compression, (c) after combined deformation, and $(\mathrm{d})$ after combined deformation and shape recovery.

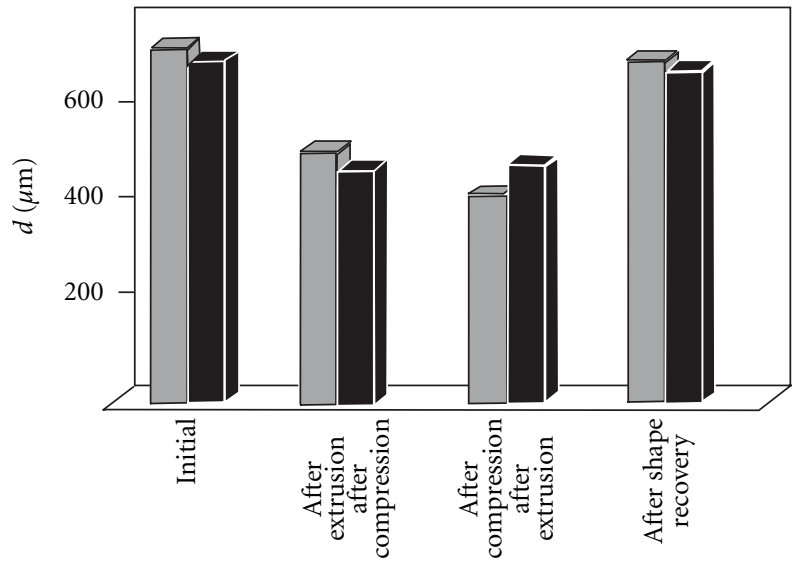

(a)

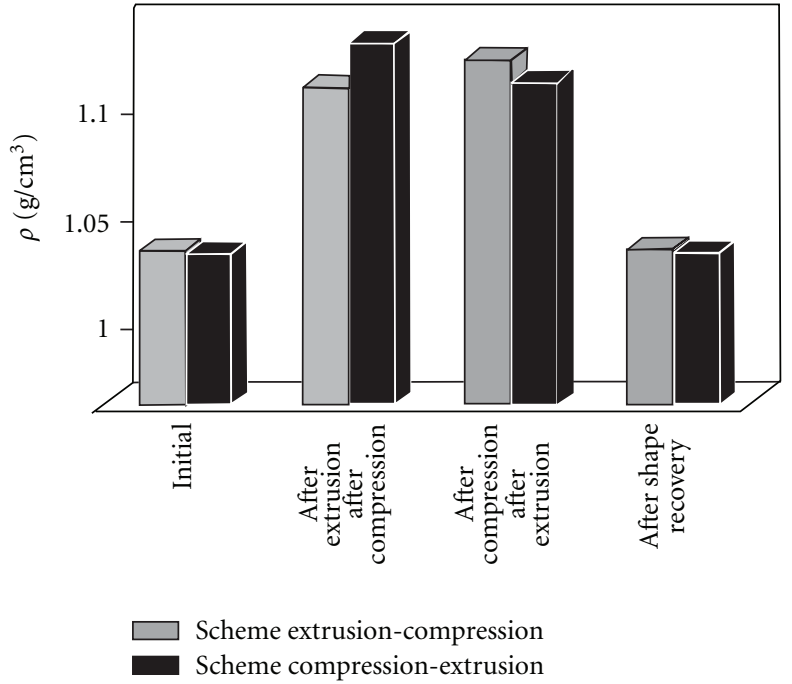

(b)

FIGURE 9: Diagrams of changes in the average size of pores filled with TEG (a) and in the density of EP-TEG composite (b) under combined deformation. 
TABLE 4: Changes in the density of samples $\left(\mathrm{g} / \mathrm{cm}^{3}\right)$ after each deformation stage for the EP-aerosil composites.

\begin{tabular}{lcccccrr}
\hline \multirow{2}{*}{ Composite } & \multicolumn{3}{c}{ Extrusion compression scheme } & \multicolumn{4}{c}{ Compression-extrusion scheme } \\
& $\theta_{c}^{\text {init }}$ & $\theta_{c}^{e}$ & $\theta_{c}^{c}$ & $\theta_{c}^{\text {rec }}$ & $\theta_{c}^{\text {init }}$ & $\theta_{c}^{c}$ & $\theta_{c}^{e}$ \\
\hline EP-10 wt.\% A-175 & 1.129 & 1.168 & 1.171 & 1.137 & 1.134 & $\theta_{c}^{\text {rec }}$ \\
EP-10 wt.\% A-300 & 1.141 & 1.184 & 1.190 & 1.144 & 1.143 & 1.194 & 1.175 \\
EP-10 wt.\% A-380 & 1.179 & 1.229 & 1.235 & 1.182 & 1.181 & 1.240 & 1.234 \\
\hline
\end{tabular}

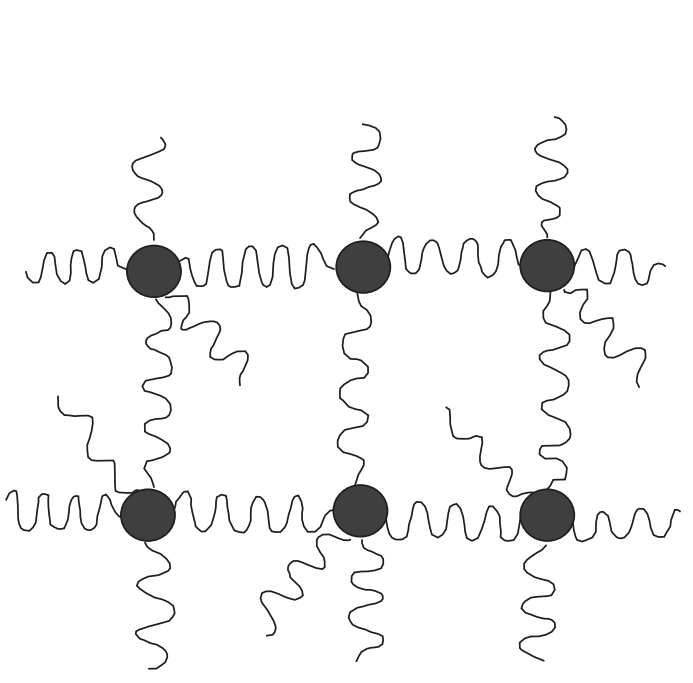

(a)

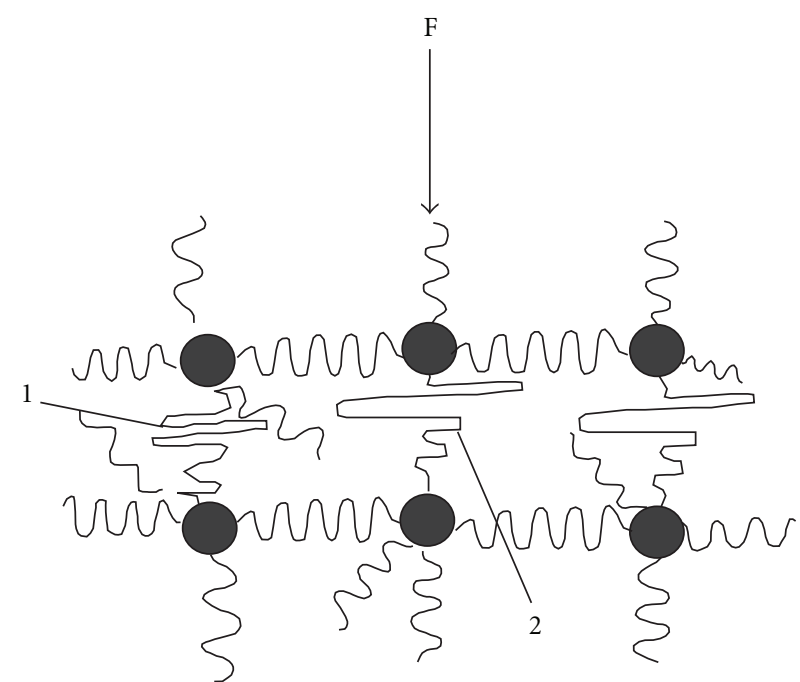

(b)

FIGURE 10: Scheme of polymer net in the initial state (a) and after compression (b).

Macromolecular structure stability is defined by the chain conformation and the force of interaction between molecular segments. Partially stretched conformations typical of the oriented polymers stipulate a state thermodynamically less stable as compared to nonoriented polymers.

For the deformed polymers the process of shape recovery is based on the tendency of attaining a thermodynamic equilibrium. The latter is defined by the minimal value of corresponding thermodynamic potential which is the difference between the internal energy and the entropy of components of macromolecules the polymer system consist of [2]. The deformation-induced orientation of polymer chains decreases the entropy of the system. Therefore the stability of deformed-polymer shape will depend on changes in the internal energy. In the amorphous polymers, the nonequilibrium state induced by deformation is only preserved after cooling below $T_{g}$. The heating above $T_{g}$ results in a growing mobility of macromolecular links which, in turn, results in conformation changes and creation of the equilibrium folded chains together with the reduction of molecular orientation.

From the topological viewpoint, a crosslinked polymer is a system of densely crosslinked and closely packed aggregates bound, as a rule, by more sparse, in view of concentration, intersite chains [9]. Apart from the latter, in the polymer there are small linear and branched molecules (sol-fraction) not relating to the net [9]. The mechanism of the crosslinked polymer deformation can be represented as follows. When the crosslinked polymer is deformed by compression, the intersite chains become folded in the mould, their axis coincides with or is at an angle to the direction of compression (chains 1,2, Figure 10). The degree of their compression depends on value of free volume of such polymer. At a definite degree of deformation, the short or completely compressed chains of type 1,2 break and there occurs polymer-net mechanical destruction.

In the case of extrusion, the intersite chains undergo tension in direction of drawing (chains 1,2, Figure 11) and compression in perpendicular direction (chains 3,4, Figure 11). For definite degrees of drawing, the short or stretched chains can also provoke the mechanical destruction of the polymer net.

Proceeding from [9], it can be assumed that in crosslinked polymers, the same as in the case of linear amorphous polymers, the deformation gives rise to microvolumes where shear deformations are localized. For high $\varepsilon$ values or with $T \geq T_{g}$ the interaction of microvolumes results in molecular rearrangement and conformation changes in the deformed chains. As a result, under heating above $T_{g}$ is observed the relaxation of molecular orientation.

To conceive changes occurring during orientation of the filled crosslinked polymer, its space structure need be 


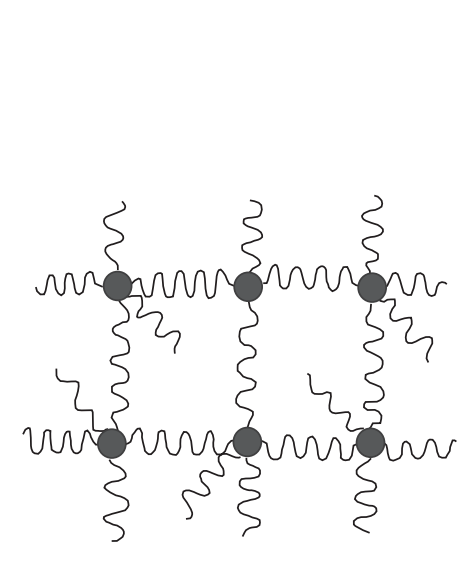

(a)

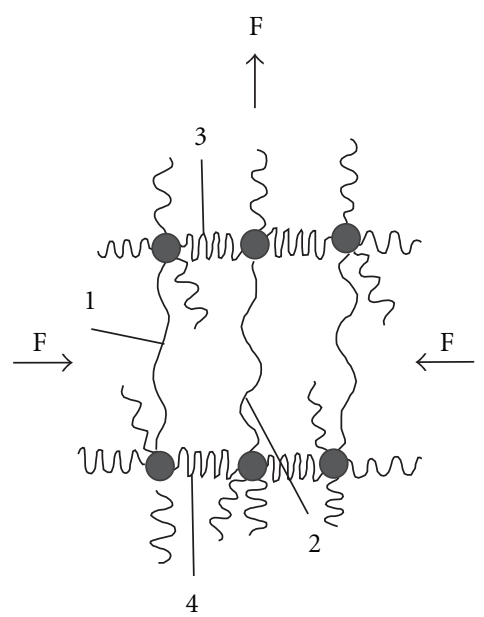

(b)

FIGURE 11: Scheme of polymer net in the initial state (a) and after extrusion (b).

simulated in view of filler distribution in the polymer in the form compactable aggregates. In the case of compression in a mould or under extrusion, the filler aggregates are compacted and the free volume is filled by polymer matrix. In this case, the mechanism of polymer-matrix deformation is similar to the described above for the unfilled polymer.

When the shape is completely recovered, the cycling with a small number of tests (to five) does not result in changes of the degree of recovery and the value of the maximum attainable deformation. It can be, thus, assumed that under cycling, the total volume of pores, due to the introduction of filler agglomerates, remains constant and the number of intersite chains participating in the loading-unloading cycles is large as compared to the number of broken chains.

\section{Conclusions}

(1) During the SME realization in composite based on network polymer and compacting filler, a change in volume is the larger the lower the relative density of the filler.

(2) The value of stresses developed in composites under shape recovery is defined by the hardening ability of the filler.

(3) Residual strain upon shape recovery is connected with the presence of boundary layers in polymer composite. Its magnitude depends on both the adsorption ability of the filler and the scheme of deformation.

(4) The utilization of combined deformation, consisting of the processes of compression and elongation of specimens in any sequence, makes it possible to form such states of composite structure when the ratio between radial and axial components of the strain can take zero, positive or negative values during the shape recovery.

\section{References}

[1] A. Lendlein and S. Kelch, "Shape-memory polymers," Angewandte Chemie - International Edition, vol. 41, no. 12, pp. 2035-2057, 2002.

[2] V. A. Beloshenko, V. N. Varyukhin, and Yu. V. Voznyak, "The shape memory effect in polymers," Russian Chemical Reviews, vol. 74, no. 3, pp. 265-283, 2005.

[3] C. Liu, H. Qin, and P. T. Mather, "Review of progress in shapememory polymers," Journal of Materials Chemistry, vol. 17, no. 16, pp. 1543-1558, 2007.

[4] I. A. Rousseau, "Challenges of shape memory polymers: a review of the progress toward overcoming SMP's limitations," Polymer Engineering and Science, vol. 48, no. 11, pp. 20752089, 2008.

[5] V. Skákalová, V. Lukeš, and M. Breza, "Shape memory effect of dehydrochlorinated crosslinked poly(vinyl chloride)," Macromolecular Chemistry and Physics, vol. 198, no. 10, pp. 3161-3172, 1997.

[6] V. A. Beloshenko, YA. E. Beygelzimer, A. P. Borzenko, and V. N. Varyukhin, "Shape memory effect in the epoxy polymerthermoexpanded graphite system," Composites Part A, vol. 33, no. 7, pp. 1001-1006, 2002.

[7] V. A. Beloshenko, V. N. Varyukhin, and Yu. V. Voznyak, "Electrical properties of carbon-containing epoxy compositions under shape memory effect realization," Composites Part A, vol. 36, no. 1, pp. 65-70, 2005.

[8] V. M. Kuznetsova, R. A. Yakovleva, A. F. Tereshchenko et al., "Current - conducting adhesion composition," Certificate of authorship no. 1824901, USSR, 1992.

[9] V. G. Khozin, Strengthening of Epoxy Polymers, Dom Pechati, Kazan, Russia, 2004.

[10] K. Gall, M. L. Dunn, Y. Liu, D. Finch, M. Lake, and N. A. Munshi, "Shape memory polymer nanocomposites," Acta Materialia, vol. 50, no. 20, pp. 5115-5126, 2002.

[11] K. Gall, M. L. Dunn, Y. Liu, G. Stefanic, and D. Balzar, "Internal stress storage in shape memory polymer nanocomposites," Applied Physics Letters, vol. 85, no. 2, pp. 290-292, 2004.

[12] V. A. Beloshenko, Ya. E. Beigel'zimer, V. N. Varyukhin, and Yu. V. Voznyak, "Strain hysteresis of polymers," Doklady Physical Chemistry, vol. 409, no. 1, pp. 207-209, 2006. 

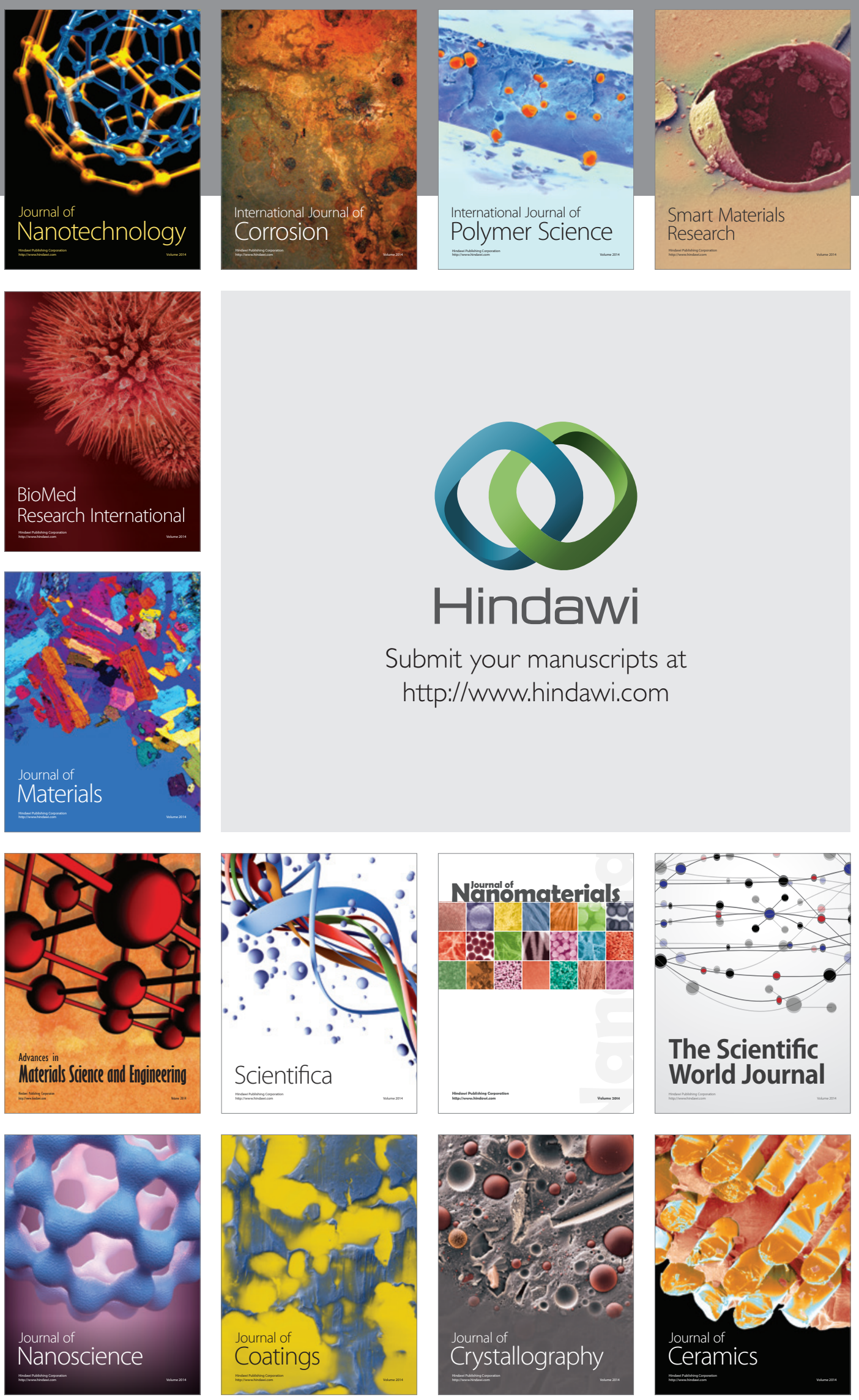

The Scientific World Journal

Submit your manuscripts at

http://www.hindawi.com

\section{World Journal}

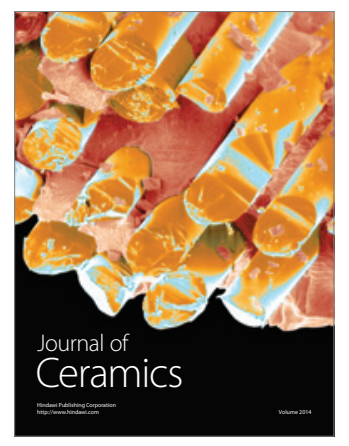

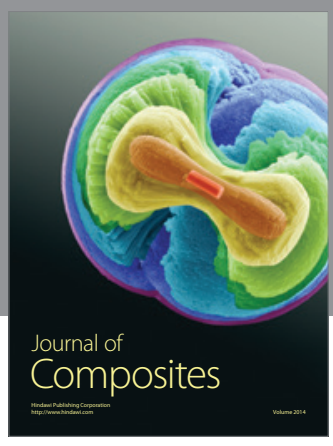
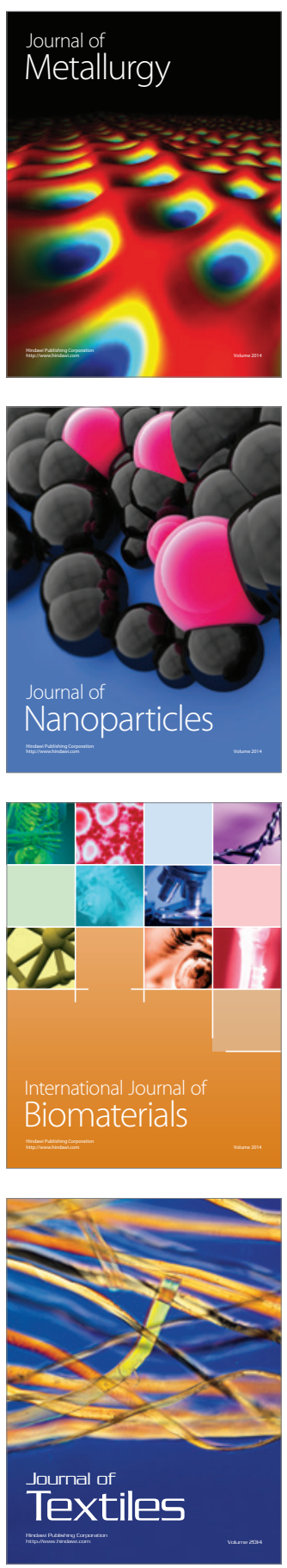